\title{
Arbeitsintensivierung - ein Merkmal der modernen Arbeitswelt?
}

\author{
ELKE AHLERS, ANNE GOEDICKE, LENA HÜNEFELD
}

$\mathrm{M}$ üssen Arbeitnehmer*innen mehr leisten als noch vor einigen Jahren? Verändern sich die Arbeitsanforderungen an Beschäftigte im Zuge des technologischen Fortschritts und vor dem Hintergrund eines intensivierten Wettbewerbs? Führen Digitalisierungsprozesse und damit verknüpfte Trends der Flexibilisierung und der Veränderung von Steuerungsformen zu Arbeitsverdichtung?

Viele Arbeitnehmer*innen und ihre Interessenvertretungen werden bei diesen Fragen zustimmend nicken. Doch ob sich diese Erfahrungen auch in einer generell beobachtbaren Entwicklung zu erhöhten Arbeitsanforderungen widerspiegeln, bleibt vonseiten der Forschung noch genauer zu klären. Gleiches gilt für die Fragen nach den Ursachen für hohe Arbeitsintensität und den Möglichkeiten, resultierende Gesundheitsrisiken für Beschäftigte zu begrenzen. Um die Arbeitswelt so zu gestalten, dass sie als menschengerecht erlebt wird und zugleich produktiv und nachhaltig sein kann, ist ein differenziertes und fundiertes Verständnis aktueller Phänomene und Wirkungszusammenhänge nötig. Dazu soll dieses Schwerpunktheft beitragen. Es versammelt Aufsätze, die sich aus unterschiedlichen konzeptionellen und empirischen Perspektiven mit der Messung und der Erklärung hoher Arbeitsintensität sowie Handlungsoptionen betrieblicher und überbetrieblicher Akteure befassen.

Verschiedene Erwerbstätigenbefragungen belegen tatsächlich die Wahrnehmung einer hohen Arbeitsintensität und verweisen dabei auf häufigen Termin-/Leistungsdruck, sehr schnelles Arbeiten oder Multitasking bei einem Großteil der Erwerbstätigen in Deutschland. Ein Trend in Richtung stetig steigender Arbeitsintensität ist mit diesen Indikatoren empirisch allerdings nicht nachzuweisen. Auch die Produktivität pro Arbeitsstunde ist, volkswirtschaftlich betrachtet, in den letzten Jahren kaum gestiegen. Reflektiert also der Eindruck vieler Arbeitnehmerinnen, dass sie immer mehr in weniger Zeit leisten müssen, nicht die Realität? Oder decken die erwähnten Indikatoren nur einen Teilbereich des Phänomens „Arbeitsintensivierung“ ab? Welche Möglichkeiten gibt es, nicht nur die Arbeitsmenge pro Zeit, sondern auch veränderte qualitative Anforderungen und eine erhöhte Komplexität von Aufgaben empirisch zu erfassen?

Teil des Problems ist, dass es in der bisherigen Forschung keine allgemeingültige Definition für Arbeitsintensität gibt. Folglich werden in Studien auch unterschiedliche Facetten betont, sodass es teilweise zu widersprüchlichen Aussagen kommt, was einer Aufarbeitung von Ursachen und Hintergründen im Wege steht. Jedoch finden sich - wie auch die Beiträge dieses Hefts zeigen - genügend Gemeinsamkeiten und Überlappungen in den Begriffsbestimmungen, um aussagekräftige Analysen vornehmen zu können.

Ausgehend von den begrifflichen Gemeinsamkeiten in der Forschungsliteratur verwenden wir in diesem Heft die Bezeichnungen „Arbeitsintensität“ und „Arbeitsintensivierung“. Der Begriff „Arbeitsintensität" beschreibt dabei das Ausmaß der Beanspruchung. Die Bezeichnung „Arbeitsintensivierung“ zielt einerseits auf eine wissenschaftliche (zeitliche) Trendaussage ab, andererseits spiegelt dieser Begriff aber auch die subjektiven Eindrücke von Beschäftigten und betrieblichen Akteuren wie Betriebsräte wider, dass immer mehr geleistet werden müsse. Eine (zu) hohe Arbeitsintensität liegt nach unserem Verständnis immer dann vor, wenn die Arbeitsmenge und die geforderte Qualität der Arbeitsleis- tung nicht zur verfügbaren Zeit für die Verrichtung der Tätigkeit passen. Hinsichtlich der Ursachen von Arbeitsintensivierung finden sich in der bisherigen Forschung sehr unterschiedliche Theorieperspektiven, etwa in Bezug auf die Frage, welcher Erklärungswert dem Handeln der Beschäftigten, der Tätigkeit, dem Arbeitsplatzumfeld und dem Betrieb oder aber überbetrieblichen Trends bzw. gesamtgesellschaftlichen Entwicklungen zukommt. Für die wissenschaftliche Analyse ebenso wie für interessenpolitisches Handeln spielt ferner eine Rolle, wie man das verbreitete „Mittun“ der Beschäftigten bei der Rationalisierung ihrer Arbeit wertet, und es gilt, positive Herausforderungen durch anspruchsvolle Arbeitsaufgaben ebenso mitzubedenken wie betriebsübergreifende, gesellschaftliche Imperative der Selbstoptimierung und Selbstrationalisierung.

Trotz vieler offener Fragen in diesem Themenfeld steht eines fest: Arbeitsintensivierung ist von hohem gesellschaftspolitischem Interesse, denn vermittelt über Erschöpfung und Erholungsunfähigkeit stellt sie ein Gesundheitsrisiko für die betroffenen Beschäftigten dar und betrifft dabei sehr unterschiedliche arbeitsweltliche Arrangements: in der Wissensarbeit, in der Krankenpflege, im Speditionsgewerbe und in vielen anderen Bereichen.

Ziel dieses Schwerpunktheftes ist es also, die vor dem Hintergrund der sich verändernden Arbeitswelt oft unspezifisch geführte gesellschaftliche Diskussion um Arbeitsintensivierung sowohl theoretisch als auch empirisch aufzuarbeiten. Dies beinhaltet, mithilfe einer interdisziplinären Herangehensweise vorliegende Kenntnisse zu Ursachen und Auswirkungen von hoher Arbeitsintensität als Belastungstrend in der modernen Arbeitswelt systematisch darzustellen, empirische Analysen zum Thema zu bündeln und theoretische Perspektiven zu kreuzen. Mit dieser theoretischen und empirischen Standortbestimmung sollen belastbare Befunde und Wissenslücken identifiziert werden. Darüber hinaus will dieses Heft für betriebliche und überbetriebliche Akteure auch Handlungsoptionen, Regulierungsbedarfe und Ideen aufzeigen sowie für die Forschung, die Politik und die betriebliche Praxis neue kritische Fragen aufwerfen. Die Debatte um Arbeitsintensivierung wird uns im Zuge des Wandels der Arbeit weiter begleiten.

\section{KONZEPT UND KOORDINATION DES SCHWERPUNKTHEFTES}

ELKE AHLERS, Dr., Leiterin des Referats Qualität der Arbeit am WSI in der Hans-Böckler-Stiftung.

Elke-Ahlers@boeckler.de

ANNE GOEDICKE, Dr., wissenschaftliche Mitarbeiterin am Landesinstitut für Arbeitsgestaltung des Landes Nordrhein-Westfalen (LIA.nrw).

anne.goedicke@lia.nrw.de

LENA HÜNEFELD, Dr., wissenschaftliche Mitarbeiterin, Bundesanstalt für Arbeitsschutz und Arbeitsmedizin, Fachgruppe 1.2 Arbeitsweltberichterstattung.

Huenefeld.Lena@baua.bund.de 